\title{
BOURDIEU AVEC FOUCAULT, UN RENDEZ-VOUS MANCATO? LE PERIPEZIE DELLO STATO TRA FOUCAULT E BOURDIEU
}

\author{
BOURDIEU COM FOUCAULT, UM RENDEZ-VOUS PERDIDO? AS VICISSITUDES \\ DO ESTADO ENTRE FOUCAULT E BOURDIEU
}

\section{BOURDIEU WITH FOUCAULT, A LOST RENDEZ-VOUS? THE VICISSITUDES OF STATE BETWEEN FOUCAULT AND BOURDIEU}

Clara Mogno

clara.mogno@hotmail.it

Dottoressa di ricerca in filosofia presso Università degli Studi di Padova. Padova, Itália.

\begin{abstract}
RIASSUNTO
Tradizionalmente, lo Stato è uno degli oggetti principali della teoria politica, della filosofia politica e della sociologia. Bourdieu e Foucault hanno entrambi tentato di comprendere lo stato e le sue funzioni, affrontando l'argomento in due modi molto diversi. Certamente condividono la volontà di analizzarlo attraverso le sue azioni e pratiche concrete: è possibile leggere una ridefinizione dello sguardo che deve essere adottato per gestire questo particolare oggetto. Dopo aver analizzato brevemente come Bourdieu definisce lo Stato, questo lavoro si concentra su tre momenti specifici in cui Bourdieu si riferisce ai lavori di Foucault durante le conferenze sullo stato che ha dato al Collège de France.
\end{abstract}

Parole chiave: Bourdieu; Foucault; Governamentality; Potere; Stato.

\section{RESUMO}

Tradicionalmente, o Estado é um dos principais objetos da teoria política, da filosofia política e da sociologia. Bourdieu e Foucault tentaram compreender o Estado e suas funções, abordando o tema de duas formas muito diferentes. Eles certamente compartilham a vontade de analisá-lo através de suas ações e práticas concretas: é possível ler uma redefinição do olhar que deve ser adotado para lidar com esse objeto peculiar. Depois de analisar brevemente como Bourdieu define o Estado, este trabalho se concentra em três momentos específicos, quando Bourdieu se refere aos trabalhos de Foucault durante as palestras sobre o Estado que ele deu no Collège de France.

Palavras-chave: Bourdieu; Foucault; Governamentalidade; Poder; Estado.

\begin{abstract}
Traditionally, the State is one of the main objects of political theory, political philosophy and sociology. Bourdieu and Foucault have both attempted to understand the State and its functions, by approaching the topic in two very different ways. They certainly share the will to analyse it through its concrete actions and practices: it is possible to read a redefinition of the gaze that must be adopted in order to handle this peculiar object. After briefly analysing how Bourdieu defines the State, this work focus on three specific moments when Bourdieu refers to Foucault's works during the lectures on the State he gave at the Collège de France.
\end{abstract}

Keywords: Bourdieu; Foucault; Governamentality; Power; State.

\section{SUMÁRIO}

INTRODUZZIONE; 1 DISCUSSIONI; CONCLUSIONE; BIBLIOGRAFIA. 


\section{INTRODUZZIONE}

Esistono dei punti di convergenza tra le analisi proposte da Bourdieu e quelle da Foucault rispetto al tema dello Stato? I due autori possono ritrovarsi su alcune posizioni o lo Stato rappresenta il luogo di un rendez-vous impossibile? È a partire da questi interrogativi che ci siamo dedicati ad una rilettura dei testi bourdieusiani e foucaultiani.

\section{DISCUSSIONI}

Il tema dello Stato, analizzato da un punto di vista sociologico, è molto presente nella produzione bourdieusiana e costituisce un nodo centrale del suo pensiero. Si pensi ad esempio a come Bourdieu in La Noblesse d'État proponga un parallelo tra la costruzione dello Stato moderno e l'istituzione della scuola pubblica ( $\mathrm{e}$, in particolare, con la costituzione delle grandes écoles): in questa direzione viene analizzato il modo in cui lo Stato, "banca centrale del capitale simbolico" si dà come garante della legittimità dei titoli scolastici. ${ }^{1}$ Per quanto riguarda in modo specifico la riflessione bourdieusiana sullo Stato sono centrali i tre corsi tenuti da Bourdieu al Collège de France tra il 1989-1990 e il 1991-1992. ${ }^{2}$ È proprio su queste lezioni che vorremmo qui concentrarci, analizzando prima le riflessioni e le definizioni formulate da Bourdieu per poi passare a vedere come, sempre in relazione allo Stato, l'autore contrappone i suoi lavori a quelli di Foucault: sarà infatti attraverso le critiche bourdieusiane alle analisi foucaultiane sullo Stato che ci concentreremo per delineare il confronto tra i due autori.

La prima lezione del corso Sur l'État, del 18 gennaio 1990, si apre con la definizione di Stato come "oggetto quasi impensabile". ${ }^{3}$ In che senso l'oggetto "Stato" sfuggirebbe alla comprensione? Secondo Bourdieu risulta “impensabile” se all'analisi non precede una riflessione circa il modo in cui ci avviciniamo a questo oggetto e ad un esame a proposito delle categorie

\footnotetext{
1 P. Bourdieu, La Noblesse d'État. Grandes écoles et esprit de corps, Paris, Éditions de Minuit, 1989, p.538: «Le titre scolaire est en effet la manifestation par excellence de ce qu'il faut appeler, par ce qui peut apparaître comme une étrange alliance de mots, la magie d'État : l'octroi d'un diplôme s'inscrit dans la classe des actes de certification ou de validation par lesquels une autorité officielle, agissant en mandataire de la banque centrale de crédit symbolique qu'est l'État, garantit et consacre un certain état de chose, une relation de conformité entre les mots et les choses, entre le discours et le réel [...].»

2 P. Bourdieu, Sur l'État, Paris, Éditions du Seuil, 2012; trad. it di M. Guareschi, Sullo Stato, Milano, Feltrinelli, 2013.

${ }^{3}$ P. Bourdieu, Sur l'État, op.cit., p.13.
} 
attraverso le quali lo pensiamo. In questo senso Bourdieu invita alla prudenza e suggerisce che se si vuole studiare lo Stato è necessario «stare particolarmente in guardia nei confronti di ciò che Durkheim definiva "prenozioni”, nei confronti delle idee ovvie e nei confronti della sociologia spontanea» ${ }^{4}$. Per studiare lo Stato sono allora necessarie una presa di distanza da parte dell'osservatore e la rottura «di una serie di schermature» prodotte dallo Stato stesso: il rischio che si corre infatti è quello di «applicare allo Stato un pensiero di Stato» e di «dire cose banali in quanto ci troviamo in qualche modo compenetrati da ciò che ci proponiamo di studiare». ${ }^{5}$ In che modo Bourdieu giustifica queste affermazioni? Lo Stato viene definito dall'autore come quel settore del campo del potere (che si può chiamare "campo amministrativo" o "campo della funzione pubblica”) che garantisce e produce l'ordine comune. È un "principio di ortodossia”, ossia un principio di produzione e di rappresentazione legittima del mondo sociale all'interno del quale si danno le lotte e i conflitti degli altri campi. Se lo Stato è esso stesso un "campo", lo è in un modo molto specifico: si potrebbe quasi dire che svolga la funzione di meta-campo, strutturante la possibilità - e le regole - del gioco strategico dei singoli campi. Il campo economico, intellettuale e quello artistico si relazionano tra loro conflittualmente ma seguendo le regole dello Stato: le loro lotte hanno come enjeux la determinazione della posizioni che essi devono legittimamente occupare gli uni rispetto agli altri. Afferma Bourdieu, riprendendo esplicitamente Durkheim, che lo Stato è "integrazione logica": permette che vi sia un "accordo sui terreni del disaccordo e sui modi di espressione del disaccordo". Lo Stato, questo "oggetto impensabile”, si pone come universale e come punto di vista neutro, un luogo che si dà (si fa riconoscere e viene riconosciuto) come indispensabile per avere lo stesso conflitto - e la sua regolazione - all'interno della società. ${ }^{6}$

Questo passaggio è riconosciuto dallo stesso Bourdieu come pericoloso, in quanto potrebbe riattivare la definizione primaria dello Stato, ossia come gli stati si autodefiniscono. Sarebbe questa la definizione che opererebbe alla base del pensiero politico moderno o, quantomeno, nel pensiero di Hobbes e Locke. Lo Stato, avendo come obiettivo principale l'organizzazione dell'ordine sociale, è preso in una continua tensione per darsi ed essere riconosciuto nel ruolo di luogo legittimo dell'espressione di un dissenso che deve, per essere espresso, accettare le regole del "gioco" statale ed esserne conforme. Lo Stato come "luogo" non

\footnotetext{
${ }^{4}$ Ibidem.

${ }^{5}$ Ivi, p.13.

6 Ivi, p.15: «Se si amplia tale definizione, si può affermare che lo Stato rappresenta la principale organizzazione del consenso inteso come adesione all'ordine sociale, ai principi fondamentali dell'ordine sociale, adesione che costituisce il fondamento non necessariamente di un consenso ma anzi della stessa esistenza degli scambi che conducono al dissenso.»
} 
può quindi essere messo in discussione: sempre secondo la teoria hobbesiana e lockiana «sarebbe un'istituzione destinata a servire il bene comune e il governo si proporrebbe di realizzare il bene del popolo.» ${ }^{7}$ È da questa visione soggiacente alla teoria classica (individuata nel pensiero filosofico-politico di Hobbes e Locke) che Bourdieu cerca di smarcarsi, ed è a partire dalla presa di distanza da questa definizione "classica" che si spiega anche la messa a critica di quella che Bourdieu chiama "sociologia spontanea dello Stato". Questa si esprime in quella che si chiama "a volte scienza amministrativa, ovvero il discorso che gli agenti di Stato producono a proposito dello Stato e che si configura come una vera e propria ideologia del servizio e del bene pubblico" . $^{\circ}$

Sempre nella stessa lezione, l'autore passa a considerare la "tradizione marxista", riferendosi in particolare ai lavori di Marx, Gramsci e di Althusser. ${ }^{9}$ Secondo Bourdieu i due autori non si interrogherebbero tanto sulla natura dello Stato, sui suoi meccanismi logici e sull'illusione ottica che le è propria ma sarebbe invece in opera nelle loro riflessioni un rovesciamento della “definizione primaria” di cui sopra. Lo Stato non sarebbe per la tradizione marxista un apparato orientato verso il bene comune ma costituirebbe invece un apparato orientato alla "coercizione, al mantenimento dell'ordine pubblico al servizio dei dominanti". ${ }^{10}$ In questo senso allora l'autore accusa la "tradizione marxista" di un'assenza di critica e di problematizzazione del meccanismo di legittimazione di sé stesso che lo Stato opera; questa tradizione sarebbe piuttosto interessata (e limiterebbe le proprie analisi) alle funzioni che esso svolge: ad un "funzionalismo del meglio" si sostituirebbe un "funzionalismo del peggio", ad un deus in machina un diabolus in machina. Divino o diabolico, orientato verso la realizzazione del bene di tutti o esclusivamente verso il bene dei dominanti, lo Stato non sarebbe in nessuna delle due tradizioni interrogato sul meccanismo logico che lo sostiene, sulla sua struttura e sulla giustificazione della sua stessa esistenza. Per Bourdieu invece deve essere indagato il modo in cui questo principio di ortodossia si dà: certo, questa finzione collettiva, questo effetto ottico di "punto di vista sui punti di vista" che produce un' "illusione ben fondata" può assolvere le funzioni che la tradizione marxista gli imputa, come quelle, ad esempio, di "conservazione sociale, di conservazione delle condizioni di accumulazione

\footnotetext{
${ }^{7}$ Ivi, p.16.

8 Ibidem.

${ }^{9}$ pp.16-17, «Da Marx a Gramsci fino ad Althusser e oltre, ci si impegna a caratterizzare lo Stato attraverso ciò che esso fa e le persone per le quali lo fa, ma senza interrogarsi sulla struttura dei meccanismi che producono ciò che fonda. E così, cis i può soffermare sulle funzioni economiche o ideologiche dello Stato parlando di "egemonia" (Gramsci) o di "apparati ideologici di Stato" (Althusser), ma l'accento cade sempre sulle funzioni, passando sotto silenzio la questione dell'essere e del fare della cosa designata con il nome di Stato.»

${ }^{10}$ p.16.
} 
del capitale". ${ }^{11}$ È d'altra parte un tema che interessa anche Bourdieu: si pensi, ad esempio, a come in La Noblesse d'État, ma anche in Les héritiers e La Reproduction, venga analizzato il modo in cui lo Stato riproduca le proprie élites e conservi l'ordine sociale attraverso l'istituzione scolastica (grazie soprattutto all'illusio del merito e attraverso il successo e l'insuccesso scolastico). ${ }^{12}$ Tuttavia, per comprendere il funzionamento dello Stato, è necessario secondo l'autore capire in primo luogo come possa costruirsi questa finzione collettiva validata dal consenso, questa "realtà misteriosa che esiste in forza dei suoi effetti e per la credenza collettiva nella sua esistenza, che costituisce il principio di quegli stessi effetti". ${ }^{13}$ Aggiunge poi Bourdieu che l'azione dello Stato è strutturante: impone schemi pratici di percezione (a questo proposito ne sono un esempio la scansione del tempo e la funzione dei calendari, categorie "cognitive reificate e naturalizzate"), categorie di classificazione e identità sociali legittime. È allora proprio a causa della presenza di queste categorie cognitive che risulta difficile pensare lo Stato, in questo senso è un "oggetto impensabile". Secondo Bourdieu, per vedere ed osservare lo Stato è necessario prenderne le distanze, smarcarsene operando un movimento di recul: le strutture stesse della coscienza attraverso le quali costruiamo il mondo sociale e l'oggetto "Stato" hanno delle buone chances di essere un prodotto dello Stato stesso. ${ }^{14}$ Si potrebbe dire che Bourdieu stia cercando un "punto di vista" autonomo e distante dal "punto di vista sui punti di vista" - e per compiere quest'operazione ermeneutica, invece che partire dallo Stato compreso come un'entità trascendente, per “échapper à la théologie", ${ }^{15}$ come dice Bourdieu, sarà allora necessario analizzare gli atti di Stato, cioè quegli "atti politici legittimi che devono la loro efficacia alla loro legittimità ed alla credenza nell'esistenza del principio che li fonda". ${ }^{16}$ Si ha qui quindi un passaggio dall'analisi del soggetto/oggetto Stato alle azioni concrete di questo: è in questa direzione che devono dunque essere considerate le commissioni statali, alle quali l'autore si dedica in queste lezioni del 1990. L'analisi delle commissioni statali - di cui Bourdieu dice essere possibile rintracciarne la genealogia ${ }^{17}$ - come la commissione Barre per il logement rientra dunque nel tipo atti di Stato che secondo l'autore devono essere il punto di partenza per un'osservazione

\footnotetext{
11 p.18.

12 P. Bourdieu, J-C. Passeron, Les héritiers. Les étudiants et la culture, Paris, Éditions de Minuit, 1964 ; P. Bourdieu, J-C. Passeron, La Reproduction. Éléments pour une théorie du système d'enseignement, Paris, Éditions de Minuit, 1970.

13 Ivi, p.24.

14 Ivi p. 13.

15 Ivi p. 24.

16 Ibidem.

17 Ivi, p.47
} 
di quest'ultimo. Sembrerebbe che Bourdieu voglia rivendicare qui una posizione empirista: passando dal soggetto/oggetto Stato (troppo astratto) alle azioni concrete che vengono fatte in nome di questo si supplisce ad una mancanza della teoria dello Stato, ovvero una necessaria osservazione della "connessione con le cose del mondo reale, della vita quotidiana" ${ }^{18}$. Alle proposizioni teologiche che considerano lo Stato come soggetto proposizionale (vero oggetto teologico, perché fondato sulla credenza), Bourdieu sostituisce allora un'analisi dei suoi atti. ${ }^{19}$

Torniamo però all'oggetto/soggetto "Stato". Abbiamo detto che questo viene definito da Bourdieu come "un principio di produzione, di rappresentazione legittima del mondo sociale". ${ }^{20} \dot{\mathrm{E}}$ sempre nella prima lezione del corso del 1990 che Bourdieu ne propone una prima definizione, ampliando la formulazione weberiana: non semplicemente "monopolio della violenza legittima" (come Weber), e nemmeno "monopolio della violenza fisica e simbolica" (come da lui proposto nell'articolo Sur le pouvoir symbolique) ${ }^{21}$ ma «"monopolio della violenza simbolica legittima", nella misura in cui il monopolio della violenza simbolica costituisce la condizione per il possesso dell'esercizio del monopolio della stessa violenza fisica. ${ }^{22}$

Ma questa definizione è, a detta di Bourdieu, ancora provvisoria. È allora scopo del corso è correggerla e precisarla e, soprattutto, analizzare in che modo e con quali strategie lo Stato si assicuri questo monopolio. Il punto è capire come lo Stato si costruisca come meta-campo, quali strategie operi per farsi riconoscere come universale, come concentri in sé la possibilità di esercizio della violenza simbolica, ovvero la possibilità di definire ciò che è legittimo e ciò che non lo è. Con violenza simbolica infatti l'autore definisce la capacità di dissimulare l'arbitrario nelle produzioni simboliche e farle ammettere come pienamente giustificate, legittime e legittimate. In questo senso lo Stato è allora il "geometrale di tutte le prospettive"23: per darsi come principio di ortodossia, come punto di vista sui punti di vista, per ottenere questo effetto di de particolarizzazione, lo Stato deve allora dare lo spettacolo dell'universale e teatralizzare l'ufficiale.

\footnotetext{
18 Ivi, p.46

19 Ivi, p.24, «In sintesi, quello che vorrei dire è: attenzione, tutte le frasi che assumono lo Stato come soggetto hanno un carattere teologico. Ciò non significa che siano false, nella misura in cui lo Stato è un'entità teologica, ossia un'entità che esiste in forza della credenza.»

${ }^{20}$ Ivi, p.14.

${ }^{21}$ P. Bourdieu, Sur le pouvoir symbolique, in “Annales”, n.3, maggio-giugno 1977.

22 P. Bourdieu, Stato, op.cit., p.14.

${ }^{23}$ Ivi, p. 53 : «rinforza un punto di vista tra gli altri sul mondo sociale, che è il luogo di lotta tra i punti di vista. Dice di questo punto di vista che è il buon punto di vista, il punto di vista dei punti di vista, il "geometrale di tutte le prospettive". E per fare questo deve far credere che lui stesso non è un punto di vista.»
} 
Vorrei ora passare ai momenti in cui Bourdieu cita Foucault in maniera esplicita durante le lezioni dei corsi sullo Stato. La prima occasione in cui Foucault viene nominato è il 15 febbraio 1990: riflettendo a proposito delle gerarchie all'interno del campo accademico e prendendo come esempio il primo numero della rivista di geografia Hérodote, Bourdieu pone l'attenzione a come la filosofia sia riconosciuta come gerarchicamente superiore alle discipline storiche e geografiche. In questo senso Bourdieu spiega la presenza di un'intervista a Foucault contenuta in quel numero: la filosofia ha un dominio simbolico sulle altre due discipline e in questo senso il "basso" chiede e trova legittimità facendo intervenire un'esponente dell' “alto". ${ }^{24}$

Il secondo riferimento esplicito a Foucault occorre nel secondo corso sullo Stato (dell'anno accademico 1990-1991). In un passaggio della lezione del 10 gennaio 1991 Bourdieu afferma che «solo la ricerca genetica ci può ricordare che lo Stato, e tutto ciò che ne segue, è un'invenzione storica, un artefatto storico e che noi stessi siamo delle invenzioni di Stato", ribadendo poi che anche il modo in cui pensiamo e le categorie che utilizziamo («i nostri spiriti») sono determinati dallo Stato, riferendosi all'azione strutturante di cui abbiamo parlato sopra). La ricerca genetica - e la sociologia come strutturalismo genetico - vengono qui apertamente contrapposte alla ricerca genealogica e nello specifico alla ricerca foucaultiana. «Fare una storia genetica dello Stato, e non una "genealogia" nel senso di Foucault, è il solo vero antidoto a quello che chiamo "l'amnesia della genesi"», oblio caratteristico di ogni «istituzionalizzazione riuscita». ${ }^{25}$ È un richiamo alla determinazione storica dello Stato, alla sua contingenza e alla sua artificialità al fine di congiurare una perfetta universalizzazione (e giustificazione) della forma Stato.

Passiamo ora a Foucault. L'autore di Sorvegliare e Punire rileva una sopravvalutazione dello Stato per proporre invece un'analitica dei poteri: è l'insieme dei poteri, e la sua complessità, che diventano gli oggetti principe d'indagine. ${ }^{26}$ Come giustamente osserva anche Arnault Skornicki in La grande soif de l'État, questo non significa che il tema dello Stato non interessi a Foucault

\footnotetext{
${ }^{24}$ Ivi, p.151, «Gli storici e, a fortiori, i geografi sono vittime di una forma di dominio simbolico che consiste nell'essere, allo stesso tempo, vittime e carnefici. Vi propongo un esempio: nel primo numero di una rivista di geografia che manifesta notevoli pretese, "Hérodote", è pubblicata un'intervista a Michel Foucault. Si tratta di un lapsus significativo: il basso chiede legittimazione all'alto."

25 P. Bourdieu, Sur l'État. Cours au Collège de France 1989-1992, Paris, Seuil, 2012, p. 185, " Aussi, seule la recherche génétique peut nous rappeler que l'État, et tout ce qui s'ensuit, est une invention historique, un artefact historique et que nous sommes nous-mêmes des inventions d'État, que nos esprits sont des inventions d'État. Faire une histoire génétique de l'État, et non pas une " généalogie » au sens de Foucault, est le seul antidote véritable à ce que j'appelle l' "amnésie de la genèse » qui est inhérente à toute institutionnalisation réussie, toute institution qui réussit à s'imposer impliquant l'oubli de sa genèse. "

${ }^{26} \mathrm{Cfr}$. A. Skornicki in La grande soif de l'État. Michel Foucault avec les sciences sociales, Paris, Éditions Les prairies ordinaires, 2015.
} 
ma che gli interessi in un modo che lui stesso definisce "differenziale". ${ }^{27}$ Una riduzione del problema dei rapporti di potere alla sola figura dello Stato, privilegiando le analisi sugli appareils d'État, non porterebbe a comprendere il funzionamento del potere ad un livello regionale: lo Stato quindi è destituito da un ruolo preminente nell'analisi da Foucault: mantiene comunque una dimensione importante ma il focus passa dall'interesse per la nascita dello Stato e per la statalizzazione della società alla governamentalizzazione dello Stato. ${ }^{28}$

Che cosa pensa Foucault rispetto alla possibilità di formulare una genealogia dello Stato moderno? Troviamo una possibile risposta a questa domanda in un passaggio importante del corso Sicurezza, territorio e popolazione, quando Foucault afferma che possibile, a condizione però di non partire «da un'ontologia circolare dello Stato». ${ }^{29}$ Se si considera infatti lo Stato come auto affermantesi e crescente come un "enorme mostro o una macchina automatica" si rischia in primo luogo di attribuire un'unità allo Stato e di renderlo una figura mitica ed astratta, di renderlo una realtà trascendente e di perdere la complessità delle costellazioni di potere, che certamente non si esauriscano nell'organizzazione statale. Genealogia dello Stato sì in Foucault, ma solo a partire (e inserendola in) da una storia della ragione governamentale: infatti obiettivo del corso è di mostrare come sia possibile, partendo da un'analisi locale e microscopica delle forme del potere che caratterizzano il pastorato, ricongiungersi ai problemi generali che sono quelli dello Stato. ${ }^{30}$

Mi sembra che il punto in comune tra Bourdieu e Foucault sia che entrambi si pongono come obiettivo di smarcarsi da un'analisi dell'oggetto Stato che lo comprenda come un'entità trascendente e universale: si tratta di mettere in questione l'evidenza di questo oggetto smarcandosi però e da un'analisi che si limiti ad una descrizione (o ad una valutazione) funzionalistica. Entrambi sottolineano in diversi modi la storicità che contraddistingue questa figura, contestando le analisi che lo assumono come universale e come astratto, l'uno attraverso

\footnotetext{
${ }^{27}$ M. Foucault, Dits et Ecrits I, “Les Réponses du philosophe", $\mathrm{n}^{\circ} 163$, p. 1680. «Il est vrai que l'Etat m'intéresse, mais il ne m'intéresse que différentiellement. Je ne crois pas que l'ensemble des pouvoirs qui sont exercés à l'intérieur d'une société - et qui assurent dans cette société l'hégémonie d'une classe, d'une élite ou d'une caste, - se résume entièrement au système de l'Etat. L'Etat, avec ses grands appareils judiciaires, militaires et autres, représente seulement la garantie, l'armature de tout un réseau de pouvoirs qui passe par d'autres canaux, différents de ces voies principales. Mon problème est d'effectuer une analyse différentielle des différents niveaux de pouvoir dans la société. Par conséquent, l'Etat y occupe une place importante, mais non pas prééminente.»

${ }^{28}$ M. Foucault, Sicurezza, territorio e popolazione, pp.88-89 e in particolare: «Lo Stato - non più oggi che nel corso della sua storia - non ha mai avuto questa unità, questa individualità, questa funzionalità rigorosa e direi anche questa importanza. Dopo tutto, forse, lo stato non è che una realtà composita e un'astrazione mitizzata la cui importanza è molto più circoscritta di quel che si crede. Ciò che è importante per la nostra modernità, cioè per la nostra attualità, non è tanto la statalizzazione della società, ma quella che chiamerei la "governamentalizzazione" dello stato."

29 Ivi, p.258.

${ }^{30} \mathrm{Cfr}$. Ivi, p.262.
} 
l'appello ad una genetica e l'altro attraverso la ricerca genealogica. In Bourdieu però, mi pare che, se da una parte è presente il riconoscimento dello Stato come un qualcosa che si dà storicamente - e di conseguenza una sua de-universalizzazione - dall'altra è presente proprio quell'ontologia circolare dalla quale mette in guardia Foucault. Benché vi sia un'analisi dell'insieme dei poteri in Bourdieu e che questi non si esauriscano nello Stato, quest'ultimo, essendo posto come il loro garante, sembrerebbe avere una posizione preminente. In questa direzione penso ad esempio a come venga affrontato il tema del monopolio della violenza simbolica dello Stato: questo è al tempo stesso ciò che definisce lo Stato e l'oggetto della sua azione. Per dirla più semplicemente, lo Stato fonda il potere simbolico $e$ si afferma attraverso di esso, si costituisce cioè per mezzo di un “colpo di Stato”, ossia accumulando potere simbolico, presentandosi, di conseguenza, come un già dato (come un soggetto d'azione) prima del suo darsi.

Vorrei ora passare velocemente ad un altro testo e poi tornare di nuovo al corso di Bourdieu. Nel 1990, il sociologo rilascia un'intervista (per France Culture) con Antoine Spire, Pascale Casanova e Miguel Bennassayag, trascritta in Si le monde social m'est supportable, c'est parce que je peux m'indigner. E a proposito del funzionamento del potere e di come si organizza la dominazione sociale Bourdieu risponde:

Quando i filosofi parlano di potere, lo cercano da qualche parte, pensano sempre allo Stato, a dei luoghi in cui il potere è incarnato, mentre il potere è inafferrabile, è ovunque e da nessuna parte, il che non vuol dire che non sia piuttosto in certi luoghi che in altri - in una struttura, ci sono dei luoghi centrali. E quindi, fare una genealogia, come dice, della dominazione, è cercare il potere sia nei meccanismi sociali che producono delle strutture ed anche nella testa degli individui. [...] Queste strutture non possono funzionare se non con la complicità degli agenti che hanno interiorizzato le strutture secondo le quali il mondo è organizzato. [...] Si dice: "Abbasso lo Stato!". Bene, lo Stato non agisce se non con ciò che ha messo di lui stesso nel nostro cervello, e vi è dunque una sorta di psicanalisi dello spirito umano che è la condizione di una lotta organizzata. Diciamo che una lotta politica organizzata comincia da sé stessi. Siamo, attraverso questo habitus, attraverso questa storia incorporata, sempre esposti a essere complici di coercizioni che si esercitano su di noi, a collaborare alla nostra propria dominazione. ${ }^{31}$

È curioso notare che in questa occasione Bourdieu utilizzi il termine genealogia e non genetica, come d'altra parte fa anche in altri momenti del corso Sur l'Etat. Per esempio, e cito dal primo corso, Bourdieu chiede "come è possibile traccia una genealogia storica di ciò che si è soliti chiamare Stato?". ${ }^{32}$ Condizione della lotta politica organizzata è il movimento di auto-

${ }^{31} \mathrm{P}$. Bourdieu, "Si le monde social m'est supportable, c'est parce que je peux m'indigner". Entretien avec Antoine Spire, Paris, Éditions de l'Aube, 2002, p.19, traduzione mia.

32 P. Bourdieu, Stato, op.cit, p.67. Si veda anche pp.94-95. 
riflessione che invita a fare Bourdieu: la presa di distanza dall' "oggetto impensabile" è in primo luogo un movimento di recul dal nostro stesso modo di pensare e dalle categorie che utilizziamo, l'analisi che deve essere fatta circa la violenza simbolica di cui lo Stato detiene il monopolio passa in primo luogo, e necessariamente, attraverso una presa di distanza da sé.

Tornando al corso bourdieusiano, vorrei ora analizzare la terza occasione in cui viene citato Foucault. Siamo all'altezza della lezione del 12 dicembre 1991 e, in questo passaggio, Bourdieu esprime la propria insofferenza rispetto a quelle che chiama le "théories éliasofoucaldeinnes”. Queste si focalizzerebbero unicamente sull'aspetto disciplinare dello Stato: ricordando e sottolineando esclusivamente l'aspetto di domesticazione dimenticano di considerare l'integrazione “dei dominati". La costruzione dello Stato avviene attraverso l'assistenza e la filantropia, l'integrazione è un movimento verso il centro, è la partecipazione all'illusione, cioè al gioco universalista dello Stato. ${ }^{33}$

Ma cosa sarebbe se non precisamente di "l'integrazione" quando Foucault pensa alla disciplina? Vorrei riprendere qui un passaggio di La société punitive, il corso tenuto da Foucault nel 1972 al Collége de France. Nelle pagine che precedono il passaggio sul quale vorrei focalizzarmi, Foucault si dedica a delineare le differenze tra quelli che chiama strumenti di soustraction (di sostrazione) e di sequestration (sequestro)

In tutti questi casi, la funzione dell'apparato rispetto alla marginalità è completamente diversa dal sistema uniforme dell'internamento classico : non si tratta affatto di emarginare, ma di fissare all'interno di un certo sistema di trasmissione del sapere, di normlizzazione, di produzione. [...] La macchina lavora per demarginalizzare e l'emarginazione non è che un effetto laterale. ${ }^{34}$

Assistenza e filantropia, per come si sono costruite cos'altro sarebbero se non pratiche di integrazione governamentali che si danno nella società disciplinare, caratterizzata dall'examen,

33 P. Bourdieu, Sur l'État, Paris, Éditions du Seuil, 2012, p.566. «Les théories éliaso-foucaldeinnes m'enervent un peu parce qu'elles retiennent uniquement l'aspect discipline de l'État. Or l'État ne marcherait pas du tout s'il était seulement domestication: il est aussi assistance, philantrophie, etc. Construire la nation, costruire l'État, costruire la nation à partir de l'État, c'est favoriser l' "integration " des dominés. Intégration : voilà encore un de ces mots qui a été beaucoup employé dans des contextes politiques différents, qui resurgit aujourd'hui, mais qui veut dire deux choses. C'est un mouvement vers le centre, c'est une partecipation à l'illusio (entrer dans le jeu) et, en même temps, intégration s'oppose à sécession, au fait de sortir de l'État. L'un des choses que l'on oublie, qui resurgit par exemple lorsque les mouvements contre l'État prennent la forme nationale, c'est qu'une des alternatives dans les luttes est l'alternative de l'intégration/assimilation et de la sécession, la sécession pouvant prendre la frome d'une rupture.»

${ }^{34}$ M. Foucault, La société punitive. Cours au Collège de France (1972-1973), Paris, EHESS, 2013; trad.it di P. Rovatti, La società punitiva. Corso al College de France (1972-1973), Milano, Feltrinelli, 2016, p. 224, 225. 
dalla prova permanente, dalla moralizzazione della classe operaia, dal libretto di risparmio, dall'insegnamento alla previdenza, dalle tecnologie di responsabilizzazione, per un intervento sugli illegalismi? Ad un certo punto non si tratta più di mettere al bando, di esiliare, marginalizzare le soggettività ma di correggerle, normalizzarle, educarle e fissarle. E soprattutto, per Foucault, quello che diventa essenziale e centrale è la fabbricazione del sociale. Questo può essere fatto attraverso le istituzioni di normalizzazione, attraverso la creazione di un'immagine della società e attraverso la creazione di una norma sociale. Riprendo qui sempre da La société punitive:

L'istituzione di sequestro [...] ha la funzione di fabbricare il sociale. Tra le classi in cui funzionano questi sistemi di sequestro e lo Stato a cui si appoggiano, questi sistemi hanno la funzione, tra l'altro, di costituire un'immagine della società, una norma sociale. Le istituzioni di sequestro fabbricano qualcosa che è al tempo stesso interdetto, norme e che deve diventare realt: sono istituzioni di normalizzazione. ${ }^{35}$

\section{CONCLUSIONE}

Per concludere. In entrambi gli autori è presente l'esigenza di uscire da una rappresentazione trascendente dello Stato ma il movimento che operano è molto differente: né Bourdieu né Foucault localizzano il potere "nello Stato" ma il primo gli assegna un ruolo preminente, paradossalmente, cadendo, a mio avviso, in quell'ontologia dello Stato di cui parla Foucault. È d'altra parte di Bourdieu l'affermazione che «lo Stato è una finzione giuridica prodotta da giuristi che si sono prodotti in quanto tali producendo lo Stato ${ }^{36}$. Quest'ultimo invece, ricorrendo alla figura del governo e intendendolo come una peripezia di quest'ultimo, lo coglie nella sua specificità di concrezione di esercizio di potere. A questo proposito, e riflettendo circa la “localizzazione del potere” in un passaggio del corso del '72-'73 afferma Foucault:

Ora, non credo che il potere possa essere descritto in maniera adeguata come qualcosa che sarebbe localizzato negli apparati di Stato. E non basta nemmeno dire che gli apparati di Stato sono la posta in gioco di una lotta, interna o esterna. Mi sembra piuttosto che l'apparato di Stato sia una forma concentrata, o comunque una struttura d'appoggio , di un sistema di potere che va ben oltre e più in profondità. Questo fa si che in pratica, né il controllo né la distruzione dell'apparato di Stato siano sufficienti a trasformare o a far scomparire un certo tipo di potere, quello in cui ha funzionato. ${ }^{37}$

\footnotetext{
35 Ivi, p.231.

36 P. Bourdieu, Stato, op.cit, p.95.

${ }^{37}$ M. Foucault, La società punitiva, op. cit., p.245.
} 
Il ricorso allo studio della ragione governamentale allora sembrerebbe essere una presa di distanza dall'oggetto che, venendo relativizzato, sembrerebbe essere più afferrabile.

\section{BIBLIOGRAFIA}

BOURDIEU, P. "Si le monde social m'est supportable, c'est parce que je peux m'indigner". Entretien avec Antoine Spire. Paris: Éditions de l’Aube, 2002.

BOURDIEU, P. La Noblesse d'État. Grandes écoles et esprit de corps. Paris: Éditions de Minuit, 1989.

BOURDIEU, P. Passeron, J. C. La Reproduction. Éléments pour une théorie du système d'enseignement. Paris: Éditions de Minuit, 1970.

BOURDIEU, P. Sur le pouvoir symbolique. In Annales, n.3, maggio-giugno, 1977.

BOURDIEU, P; Passeron, J. C. Les héritiers. Les étudiants et la culture. Paris: Éditions de Minuit, 1964.

FOUCAULT, M. Dits et Ecrits I. In Les Réponses du philosophe, n. 163, p. 1680.

FOUCAULT, M. La società punitiva. Corso al Collège de France (1972-1973). Milano: Feltrinelli, 2016.

SKORNICKI, A. La grande soif de l'État. Michel Foucault avec les sciences sociales. Paris: Éditions Les Prairies Ordinaires, 2015.

Artigo convidado

\section{COMO FAZER A REFERÊNCIA DO ARTIGO (ABNT):}

MOGNO, Clara. Bourdieu avec Foucault, un rendez-vous mancato? Le peripezie dello stato tra Foucault e Bourdieu. Revista Eletrônica do Curso de Direito da UFSM, Santa Maria, RS, v. 13, n. 2, p. 788-799, ago. 2018. ISSN $1981-3694$. Disponível em: < https://periodicos.ufsm.br/revistadireito/article/view/34421 >. Acesso em: dia mês. ano. doi: http://dx.doi.org/10.5902/1981369434421 . 